\title{
Research and Implementation of Service Management in Cloud
}

\section{Database}

\author{
Tao $\mathrm{Yu}^{1}$ \\ ${ }^{1}$ College of international education, Hohhot Vocational College, Hohhot, Inner Mongolia, 010051
}

KEYWORDS: Cloud Database; System Management; Super Computer

\begin{abstract}
Cloud database follow the calculation model of cloud computing that everything is a service, therefore, the service is one of the key concepts in cloud database. Because of the simplicity of the database service package, it is easy to expand for the cloud database. Cloud database provide database service for tenants through the lease delivery mode, user simply can be connect to the cloud database through the connection string, and it is easy to use obviously. How to effectively manage database service and how to guarantee the high availability, high scalability characteristics under the premise of providing services, is an important issue for cloud database to have a research.
\end{abstract}

\section{Introduction}

Cloud is a cloud database product database technology new requirements, cloud computing in database technology of advanced applications. Using a multi-tenant cloud database form to solve the problem and centralized data sharing, users pay on demand when you use a cloud database service, which greatly reduces the user cost, while effectively integrating the physical resources. In addition, users only need to use the connection string to effectively access the database in the cloud, easy to use. Theoretically, the cloud database should have unlimited scalability to meet the growing user demand for data storage, in which the changing needs of users, cloud database should be able to show a good stretch. This scalability is precisely followed the service-oriented architecture features. We followed the cloud database cloud computing "everything is service" computing model, the visible cloud database service is the core concept. How to effectively manage database services, under the premise of how to ensure high availability, high scalability, and other characteristics of external services it is an important topic cloud database requires continued research.

\section{Overall Design of Cloud Database Service Management Platform}

External cloud database service management platform to provide database services, internal use basic services. The design process need to focus on basic services and database services of these two services. Effective representation of cloud database service management platform needs to have database services and basic services, and management.

Users can lease after signing the contract cloud database service platform required to provide users with easy-to-use database service interface, service quality monitoring component, according to the user contract terms of quality of service performance assurance database services, namely, providing internet service (Platform as a Service, PaaS) operating mode JDBC interface and software as a service (software as a service, SaaS) mode of operation of Web services. Design cloud database service management platform to focus on the following three services rigging. Based 
service provider: service lifecycle management infrastructure, including basic service package, registration, deploy, start;

Database service provider, which is a cloud database service platform, provide external database services, platforms of various basic components in the form of basic services hosted service center platform as the basis for service users, bus-based calling service basis, in order to achieve database services platform; database service users: database service users and service providers of a service contract negotiated by the cloud database service interface database services;

Cloud computing model to provide database service aims to provide superior to the traditional database management system services, users only need to deploy the application interface or through the Web Interface can use database services directly. So cloud database must ensure high availability database services, in case of node failure through service scheduling, data replication and other means to ensure the continued availability of user data. Users in the use cloud database, node does not need to run the physical database management, information does not need to know the physical node, the user through a valid URL, we can use a cloud database.

The catalog records are available both inside and outside the platform of services. A typical service catalog includes service name, service levels, tester services and services dependent components, and so on. Visible, service catalog management services in complex cloud environment required.

Users in the use cloud database service need to see the cloud database service specific use, the platform should be capable of real-time monitoring service status, quality of service and resource flows, so the cloud database system needs to integrate service monitoring tools for unified management of internal services, and try to be able to show the status of these services in a visual management interface.

To solve the problem of virtual services to the effective deployment of resources and the physical configuration of all components of cloud computing applications need to design a resource dispenser. Multi-tenant database service through the application interface, cloud computing application computing cost, effort and resource scheduling component model through service, improve the performance of cloud services. Cloud computing applications currently makes extensive use of virtualization technology, this approach increases the flow of resources, while making changes to these resource monitoring becomes difficult, it is necessary to record these resources, track changes in these resources, recording their status, which requires configuration management database that stores records these changes.

\section{Cloud Database Service Management of Key Technologies}

In the study cloud database, the current multi-tenant data storage ways are: standalone database; shared database, stand-alone mode; shared database, shared model the three ways. (1) Independent mode database independent database is assigned an independent database for each tenant, each tenant individually store data, consider from the implementation point of view, this approach is simple. From the physical storage of data that in this implementation, each tenant has a separate set of data, and stored separately, and different tenants have a separate database access process on the same physical machine. This method ensures good isolation between multi-tenant, at the same time, simple data migration patterns. However, this approach cannot be caused by shared memory and database connection pool between different cloud processes, the number of the number of sets made per server is limited, this will certainly lead to a cloud database service providers to increase acquisition and maintenance costs. (2) Shared database, shared database in standalone mode, independent mode database is shared between different tenants, but the data stored in different data 
models, and each has a separate data table structure. Tenant database using the service, you need to create tenant environments in public databases, then each tenant can also create a unique table structure and data association in the respective modes. This approach makes it possible to be shared between different tenants connection pooling and memory resources are saved expenses, and for the safety requirements of tenants high a degree of logical data isolation, but it is not totally isolated. Each physical database can support a greater number of tenants. But shared among tenants with a database process, then see a different tenant database shared resource competition. (3) A shared database, shared model shared database, shared schema that is different tenants share the same database schema. Features of this approach is a data table can contain multiple tenants simultaneously record data in the table need to distinguish between individual tenants through tenant data ID. In this way, tenants can save the data is physically common table, you can distinguish different data logically tenants through tenant ID. In this way compared to the cost of the first two methods is the lowest, the number of physical database support tenant is also the largest, but also the lowest level of isolation between tenants, the lowest security, which takes a lot of work in control design and development . Data backup and recovery is also very troublesome, need to gradually restore the table. In addition, since the tenant concurrency this way a lot, and share database connection pool and memory among tenants, many tenants when the number of servers, the performance will be significantly decreased. Database management system in the cloud services and cloud database virtualization infrastructure service layer of the design process, it should be from various isolation levels, safety, design complexity, and the number of tenants and other costs are the three ways to select the appropriate multi-tenant storage solution, and then multi-tenant storage solution, properly designed surface to multi-tenant database service management.

First satisfaction algorithm is the basis for the allocation of service providers and service users between the equalization processes do not apply to purely environmental cloud database service for end users cloud services management. Reference herein First reward algorithm strategy, design service scheduling policy based on user quality of service contracts and the state of the database service. Cloud database service tenants before using the database service, on-demand service demand quality, select the service quality of service provision, the quality of the service contract signed, after the success of the service purchased. Cloud service users access the database through the application of cloud database service terminal node database configuration parameters, user parameters, contracts message, sending a service request database. Cloud database service nodes interact with other components, service scheduling issue a request to the dispatcher based on the following five strategies by the end of the service call.

Responder services require authentication of user information and contract information, and service information, user rights, and to determine the type of resource needs.

Service scheduling end resources according to the type of service parameters and parameter information came, matching resource levels to determine the type of service resources, determine available service nodes collection.

Service monitoring component for service set list each service basic state tenants quantity $\mathrm{M}$ and the physical node load $\mathrm{N}$ ( $\mathrm{N}$ is the weight physical node CPU, memory, IO number of parameters and, $\mathrm{N}=\mathrm{Ni}$, Ni denotes a CPU, weight memory usage, etc.), will be available to the service dispatch services set back end.

Service Call Select the final end service node according to Min $(0.5 * \mathrm{M}+0.5 * \mathrm{~N})$ strategy. 
Service call clients to obtain from the service registry service node information, access the database service node number, this number as a parameter service call request is sent to the service dispatcher.

It is based on the scheduler service call number pass over the end of the address Services service entities, sent to the enterprise service bus, called by the enterprise service bus implementation, service and return the results.

\section{Conclusion}

This paper focuses on the key technologies related to cloud database service management and based on open source enterprise service bus implementation platform, compliant with the current ESB design pattern, has designed and implemented to provide on-demand services, multi-tenant, high-availability, scalability, etc. characteristics of cloud database service management framework.

\section{Reference:}

[1]Hacigumus H, Iyer B, Mehrotra S. Providing database as a service. in: Proceedings of the 18th International Conference on Data Engineering. LosAlamitos, CA, USA: IEEE Computer Society, 2002. 29 38.

[2]Seibold M, Kemper A. Database as a Service. Datenbank-Spektrum, 2012, 30(2):1216 1217

[3]Ferrari E .Database as a Service: Challenges and solutions for privacy and security: 2009 IEEE Asia-Pacific Services Computing Conference( APSCC 2009). Piscataway, NJ, USA: IEEE, 2009. 46 51.

[4]Hadavi M A, Noferesti M, Jalili R, et al. Database as a Service: Towards a Unified Solution for Security Requirements. in: Proceedings of the 2012 IEEE 36th IEEE Annual Computer Software and Applications Conference Workshops (COMPSACW). Los Alamitos, CA, USA: IEEE, 2012. 415 420. 